\title{
The analgesic potency of acetaminophen, aspirin, and ibuprofen in 2- and 15-month-old rats
}

\author{
JOSEPH J. KOMAN and ROBERT J. HAMM \\ Virginia Commonwealth University, Richmond, Virginia
}

\begin{abstract}
A method developed by Randall and Selitto (1957) for testing the potency of analgesic drugs was used to examine the effectiveness of acetaminophen, aspirin, and ibuprofen in 2- and 15month-old rats. A yeast solution was injected into the rear paw of the rats to produce inflammation. To measure the degree of analgesia produced by each drug, pressure was exerted on the inflamed paw, and the pressure $(\mathrm{mmHg})$ that produced a pain response was recorded. The results of the experiment revealed that each drug produced an increased pain threshold in the younger animals, but none of the drugs were effective in the older animals.
\end{abstract}

In 1957, Randall and Selitto developed a method for testing various types of analgesic drugs. Because inflammation made the foot of a rat more susceptible to pain, Randall and Selitto constructed a device to apply pressure to an inflamed foot. Using this procedure and modifications of it, a number of experimenters (Chipkin, Latranyi, Iorio, \& Barnett 1983; Ferreira, Lorenzetti, \& Correa, 1978) have examined the analgesic potency of a number of widely used analgesic drugs including acetaminophen, acetylsalicylic acid, codeine, and morphine.

Although the analgesic potency of acetaminophen, aspirin, and ibuprofen have been previously investigated in young rats, no investigations of the analgesic potency of these drugs have been undertaken in aged animals. There are two reasons to hypothesize that there may be agerelated differences in the analgesic potency of these drugs. First, a number of investigators have observed age-related differences (both hypersensitivity and hyposensitivity) in response to other drugs that produce analgesia (e.g., Knisely \& Hamm, 1985; Lal, Gianforcaro, \& Nandy, 1979). Moreover, recent researchers have observed agerelated deficits and enhancements in some endogenous analgesic systems (e.g., Hamm \& Knisely, 1985, in press; Hamm, Knisely, \& Watson, in press). And because some exogenous analgesic drugs are thought to activate endogenous analgesic systems (Mayer, 1979), the observation of age-related differences in endogenous analgesic systems provides an additional reason to test the effect of N-Acetyl-p-Aminophenol (acetaminophen), salicylic acid (aspirin), and ibuprofen in animals of different ages.

\section{METHOD}

\section{Subjects}

Twelve 2-month-old and 6 15-month-old Sprague-Dawley rats were used. The 2-month-old animals weighed an average of $538 \mathrm{~g}$, and the

The authors thank Joseph Porter for his assistance in this project. Requests for reprints may be sent to either author, Department of Psychology, Virginia Commonwealth University, Richmond, VA 23284.
15 -month-old animals weighed an average of $590 \mathrm{~g}$. They were individually housed in an animal colony with a constant room temperature $\left(20^{\circ}-22^{\circ} \mathrm{C}\right)$ and a 6 -h light/18-h dark cycle. Food and water was continuously available to the animals in their home cages.

\section{Apparatus}

A Randall-Selitto paw test device was constructed from a Marshall Blood Pressure Kit (Model 104). The tubing between the arm cuff and pressure gauge was cut and a three-way valve was inserted. Tubing from the stethoscope was connected to the third valve and a 10-ml syringe. The syringe was positioned on the wall above the experimental table so the plunger of the syringe moved downward when pressure was applied to the system from the inflation bulb. A plastic needle cap was attached to the flat surface of the plunger of the syringe. This cap exerted the pressure on the foot of the rat.

\section{Drugs}

N-Acetyl-p-Aminophenol and ibuprofen (both from Sigma Chemical Company) were suspended in methyl cellulose. These drugs were shaken and stirred for $\mathbf{2 0}$ min prior to administration. Salicylic acid (Sigma Chemical Company) was dissolved in saline. Granular brewer's yeast was suspended in saline. Metofane (Pitman-Moore, Inc.) was used to produce general anesthesia.

\section{Procedure}

Pretesting. Two days before the experiment began, the animals became acquainted with the experimental procedure. The blood pressure cuff was filled with air until the pressure gauge read $20 \mathrm{mmHg}$. The right rear foot of the rat was placed under the plunger. The foot was placed so that the plastic needle cover would land on the center portion of the foot. The inflation bulb was squeezed so the plunger would start to fall. The plunger slowly dropped on the foot at the $80-\mathrm{mmHg}$ mark. However, the bulb was continually squeezed, thus increasing the pressure until the rat made a pain response. The pressure was increased at a rate of approximately $20 \mathrm{mmHg} / \mathrm{sec}$. A vocalization, a shake, a struggle, or a foot withdrawal was considered a pain response. The pressure that produced a pain response was recorded. Generally a pressure reading of $200 \mathrm{mmHg}$ produced a pain response from rats with uninflamed feet.

Drug testing. The animals were weighed to determine the dosage of the analgesic drugs and vehicle. The animals were lightly anesthetized with Metofane, a short-acting inhalant anesthetic, prior to receiving an injection of $0.1 \mathrm{ml}$ of a $20 \%$ suspension of brewer's yeast into the plantar surface of the right rear foot. Sixty minutes later, after the foot became inflamed, three baseline analgesic measurements were taken at 2-min intervals as described above. After the baseline analgesic measurements were taken, the animals received a drug injection. The drug dosages chosen for use in this study were the highest used by Randall and Selitto (1957). Because Randall and Selitto (1957) did not use ibuprofen, this drug was equated in strength with the dosage of acetaminophen and aspi- 
rin used. Animals from both age groups were injected subcutaneously with either acetaminophen $(100 \mathrm{mg} / \mathrm{kg})$, aspirin $(100 \mathrm{mg} / \mathrm{kg})$, ibuprofen $(30 \mathrm{mg} / \mathrm{kg})$, or an equal volume of methyl cellulose vehicle. Analgesic pressure readings were taken at 10,30 , and 60 min after drug injection. The order of drug administration was counterbalanced across animals. Drug injections were repeated at 1-day intervals for 4 days for all animals.

\section{RESULTS}

In order to determine whether the injection of yeast produced equivalently painful inflammations in each age group, the baseline pressures $(\mathrm{mmHg})$ that resulted in a pain response were analyzed using a $t$ test. This test indicated that the age groups were not significantly different. Thus, the assessment of the analgesia produced by the drugs would not be confounded by age-related differences in inflammation-induced pain.

The pressure $(\mathrm{mmHg})$ required to produce a pain response in the 2-month-old rats was analyzed by a 4 (drug) $\times 4$ (time) analysis of variance. These data are presented in Figure 1. As is illustrated in the figure, acetaminophen, aspirin, and ibuprofen were associated with an increase in pain threshold relative to the vehicle control over the 60 min test intervals. In the ANOVA, these differences in nociception were reflected in both a drug main effect $[F(3,165)=4.57, p<.0044]$ and a time main effect $[F(3,165)=4.73, p<.0036]$. The drug $\times$ time interaction was not significant. Post hoc Tukey tests were used to determine which drug conditions were

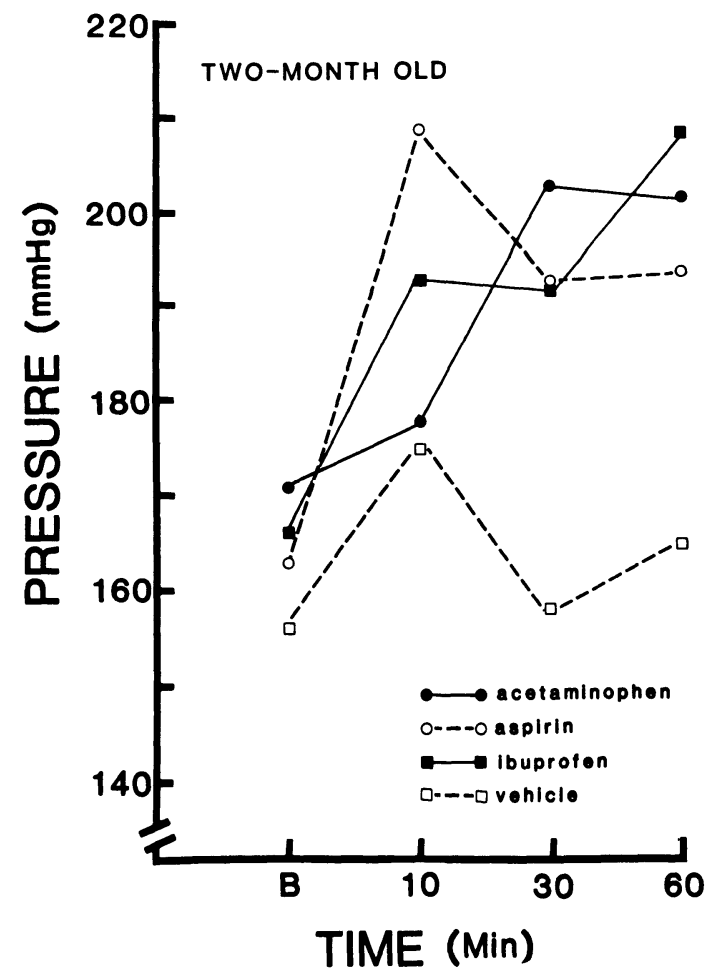

Figure 1. Mean pressure readings for 2-month-old animals. These data demonstrate that acetaminophen, aspirin, and ibuprofen produced analgesia. (B was the baseline pressure reading.)

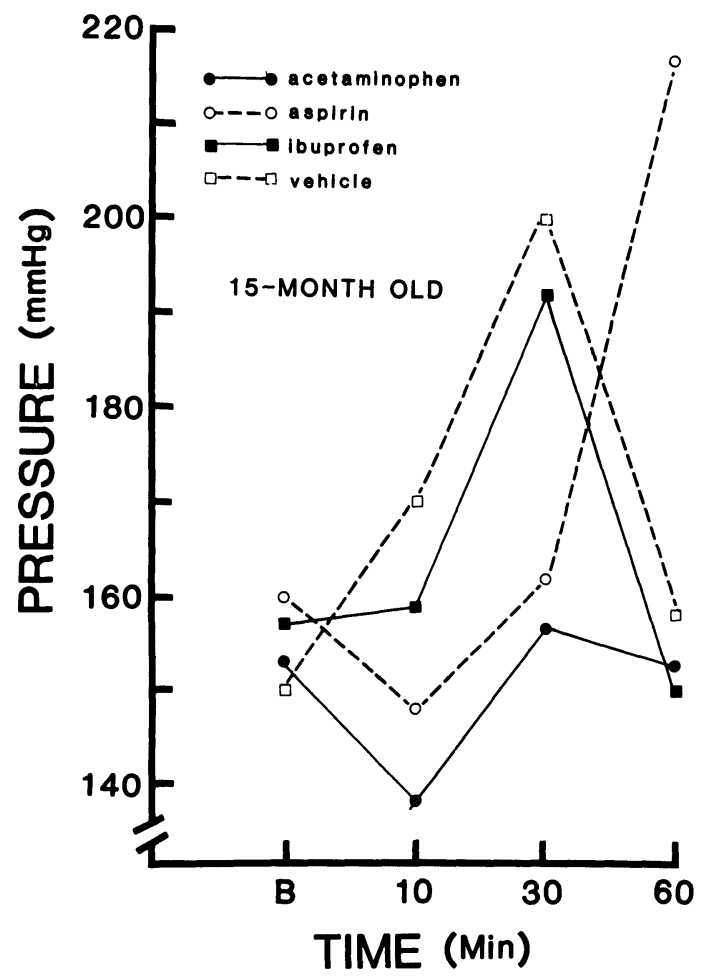

Figure 2. Mean pressure readings for 15-month-old animals. These data demonstrate that the drugs did not produce analgesia in these animals. (B was the baseline pressure reading.)

significantly different. These tests revealed that acetaminophen, aspirin, and ibuprofen were each significantly different $(p<.05)$ from the vehicle control condition, and there were no significant differences between the three drug conditions.

The pressure $(\mathrm{mmHg})$ that was required to produce a pain response in the 15-month-old rats was also analyzed by a 4 (drug) $\times 4$ (time) ANOVA. These data are presented in Figure 2. Unlike the data obtained from the two-month-old rats (Figure 1), acetaminophen, aspirin, and ibuprofen did not produce an increase in pain thresholds relative to the vehicle control condition. This conclusion was supported by the ANOVA which did not yield any significant effects (drug, time, or drug $x$ time interaction.).

The vehicle control condition did not produce stable pain thresholds over time in the 15-month-old animals in the assessment of the analgesic effectiveness of these drugs (see Figure 2). To avoid this potential problem, an additional analysis was conducted in which the predrug baseline pain thresholds were used to examine the change in pain thresholds produced by acetaminophen, aspirin, and ibuprofen. For each animal, the percent of change in baseline pain threshold was calculated at 10,30 , and $60 \mathrm{~min}$ following drug injection. These data were analyzed by a 3 (drug) $\times 3$ (time) ANOVA. The analysis yielded no significant effects. Thus, an examination of the analgesic effects of acetaminophen, aspirin, and ibuprofen relative to basal pain sensitivity also found no 
evidence for the effectiveness of these drugs in 15month-old animals.

\section{DISCUSSION}

The results of this experiment support the effectiveness of acetaminophen, aspirin, and ibuprofen in increasing the pain threshold in 2-month-old rats using the Randall-Selitto procedure. Thus, the results are in agreement with prior research (e.g., Chipkin et al., 1983; Ferreira et al., 1978; Randall \& Selitto, 1957) that has demonstrated the analgesic potency of these drugs. The results obtained from the 15month-old animals present quite a different picture. None of the drugs produced a significant increase in antinociception (measured relative to the vehicle control or basal nociception) in the 15-month-old animals. Thus, acetaminophen, aspirin, and ibuprofen were all ineffective analgesics in the older animals. This conclusion is puzzling given the widespread use of these analgesic drugs (as well as other drugs) in the human geriatric population to control arthritic and other types of pain (Ouslander, 1981; Rock, 1985; Ward \& Blatman, 1979).

There are a number of interpretations that can be applied to the results of this study. First, perhaps the Randall-Selitto procedure was an inappropriate animal model to use on an aged rat population to test the antinociceptive effectiveness of these drugs. Second, higher drug doses and longer observation times may be required to demonstrate the analgesic potency of acetaminophen, aspirin, and ibuprofen in older animals. These alternatives should be examined before conclusions can be drawn about the possibility of age-related differences in the analgesic potency of acetaminophen, aspirin, and ibuprofen.

\section{REFERENCES}

Chipkin, R. E., Latranyi, M. B., Iorio, L. C., \& Barnett, A. (1983). Determination of analgesic drug efficacies by modification of the Randall and Selitto rat yeast paw test. Journal of Pharmacological Methods, 10, 223-229.
Ferreira, S. H., Lorenzetti, B. B., \& Correa, F. M. A. (1978). Central and peripheral antialgesic action of aspirin-like drugs. European Journal of Pharmacology, 53, 39-48.

HAMM, R. J., \& KNISELY, J. S. (1985). Environmentally induced analgesia: An age-related decline in an endogenous opioid system. Journal of Gerontology, 40, 268-274.

Hamm, R. J., \& KNISELY, J. S. (in press). Environmentally induced analgesia: An age-related decline in a neurally mediated, nonopioid system. Psychology \& Aging.

Hamm, R. J., KNiSely, J. S., \& Watson, A. (in press). Environmentally induced analgesia: Age-related changes in a hormonally mediated nonopioid system. Journal of Gerontology.

KNISELY, J. S., \& HAMM, R. J. (1985). Oxotremorine-induced analgesia in mature and senescent rats. Society for Neuroscience Abstracts, 11, 731 .

Lal, H., Gianforcaro, R., \& NANDy, K. (1979). Marked alterations in responsivity to psychostimulant and cholinergic drugs associated with senescence in the female mouse. Neuroscience Abstracts, $5,7$.

MAYER, D. J. (1979). The centrifugal control of pain. In N. K.V. Ng \& J. J. Bonica (Eds.), Pain discomfort and humanitarian care. North Holland: Elsevier.

Ouslander, J. G. (1981). Drug therapy in the elderly. Annals of Internal Medicine, 95, 711-722.

Randall, L. O., \& Selitto, J. J. (1957). A method for measurement of analgesic activity on inflamed tissue. Archives Internationales de Pharmacodynamie et de Therapie, 111, 409-419.

RocK, R. C. (1985). Monitoring therapeutic drug levels in older patients. Geriatrics, 40, 75-86.

Ward, M., \& Blatman, M. (1979). Drug therapy in the elderly. American Family Physician, 19, 143-152.

(Manuscript received for publication October 21, 1985.) 